\title{
THE AUTOMORPHISM GROUP OF A FINITE METACYCLIC $p$-GROUP
}

\author{
RICHARD M. DAVITT
}

Abstract. In this paper it is shown that if $G$ is a finite nonAbelian metacyclic $p$-group, $p \neq 2$, then the order of $G$ divides the order of the automorphism group of $G$.

It is well known that if $G$ is a finite noncyclic Abelian p-group of order greater than $p^{2}$, then the order $|G|$ of $G$ divides the order of the automorphism group $A(G)$ of $G$. This result has recently been extended to other classes of finite p-groups [1], [6]. We recall that a group $G$ is said to be metacyclic if $G$ possesses a cyclic normal subgroup $K$ such that $G / K$ is also cyclic. The purpose of this paper is to show that $|G|$ divides $|A(G)|$ if $G$ is a finite noncyclic metacyclic $p$-group of order greater than $p^{2}, p \neq 2$.

The following notation is used: $G$ is a finite $p$-group where $p$ is a prime; class $G$ denotes the nilpotency class of $G ; G_{n}$ is the $n$th element in the descending central series of $G ; Z(G)$ denotes the center of $G$ (or $Z$, if no ambiguity is possible); $H \leqq G$ means $H$ is a subgroup of $G,[G: H]$ denotes the index of $H$ in $G$ and $H \triangleleft G$ means that $H$ is normal in $G$. If $x, y \in G$, then $|x|$ denotes the order of $x,(x, y)$ $=x^{-1} y^{-1} x y$ and $\langle x, y\rangle$ is the subgroup generated by $x$ and $y$; more generally, if $S$ is a subset of $G$, then $\langle S\rangle$ is the subgroup generated by $S ; P(G)=\left\langle x^{p}: x \in G\right\rangle$ and $\Omega_{m}(G)=\left\langle x \in G:|x| \leqq p^{m}\right\rangle . I(G)$ denotes the group of inner automorphisms of $G$; $I$ is the identity subgroup of $A(G)$; if $S \leqq A(G), C(S)$ is the centralizer of $S$ in $A(G)$ and $N(S)$ is the normalizer of $S$ in $A(G)$.

Before proving the main theorem of the paper, we will establish a number of preliminary results.

Lemma 1. Let $m$ and $n$ be positive integers. If $p \neq 2$, then

(i) $\left(1+p^{m}\right)^{n} \equiv 1 \bmod p^{n+m}$ and

(ii) $\left(1+p^{m}\right)^{p^{n-1}} \equiv\left(1+p^{n+m-1}\right) \bmod p^{n+m}$.

Proof. (i) Since $1+p^{m} \equiv 1 \bmod p^{m},\left(1+p^{m}\right)^{p^{n}} \equiv 1^{p^{n}} \bmod p^{n+m}[4$, Lemma 3.2 (iv)].

(ii) Part (ii) is proved by induction on $n$. The straightforward but

Received by the editors November 24, 1969.

AMS Subject Classifications. Primary 20XX; Secondary 2022, 2025, 2040.

Key Words and Phrases. Finite p-groups, regular, metacyclic, automorphism group. 
computational induction proof also uses [4, Lemma 3.2 (iv)], the binomial theorem and the fact that $p \neq 2$.

Lemma 2. Let $K \triangleleft G$ and let $a \in G$ be such that $G / K=\langle a K\rangle$ is cyclic of order $p^{n}$. If $e \neq a^{p^{m}} \in \Omega_{n}(Z)$, then the mapping $\theta\left(a, K, a^{p^{m}}\right)$ defined by $a^{j} k \theta\left(a, K, a^{p^{m}}\right)=a^{j\left(p^{m}+1\right)} k$, where $0 \leqq j<p^{n}$ and $k \in K$, is an automorphism of $G$ of order $|a| / p^{m}$ which fixes $K$ elementwise.

Proof. Let $\theta\left(a, K, a^{p^{m}}\right)=\theta$. If $g, h \in G$, then $g=a^{j_{1}} k_{1}$ and $h=a^{j_{2}} k_{2}$, where $0 \leqq j_{1}, j_{2}<p^{n}$ and $k_{1}, k_{2} \in K$. If $k_{1} a^{j_{2}}=a^{j_{2}} k_{3}$, where $k_{3} \in K$, then $g h=a^{j_{1}+j_{2}} k_{3} k_{2}=a^{j_{3}+r p^{n}} k_{3} k_{2}$, with $j_{1}+j_{2}=j_{3}+r p^{n}, 0 \leqq j_{3}<p^{n}$ and $r=0,1$. Consequently,

$$
\begin{aligned}
g h \theta & =a^{j_{3}\left(p^{m}+1\right)} a^{r p^{n}} a^{r\left(p^{m+n}\right)} k_{3} k_{2} \\
& =a^{\left(j_{3}+r p^{n}\right)\left(p^{m}+1\right)} k_{3} k_{2} \\
& =a^{j_{1}\left(p^{m}+1\right)} a^{j_{2} p^{m}} a^{j_{2}} k_{3} k_{2} .
\end{aligned}
$$

Since $a^{p^{m}} \in Z$, we see that

$$
g h \theta=a^{j_{1}\left(p^{m}+1\right)} k_{1} a^{j_{2}\left(p^{m}+1\right)} k_{2}=g \theta h \theta .
$$

Hence $\theta$ is an endomorphism of $G$.

Clearly $\theta$ fixes $K$ elementwise and since $a \theta=a^{1+p^{m}}, \theta$ is onto and hence an automorphism. Let $|a|=p^{s}$. Since $a \theta^{t}=a^{\left(1+p^{m}\right)^{t}}$ for each positive integer $t$, we see by Lemma 1 that $a \theta^{p^{-m}}=a$ while $a \theta^{p^{-m-1}} \neq a$. Hence $|\theta|=p^{s-m}$.

The definition of a regular $p$-group and the basic properties of such groups are well known and may be found in any standard group theory text (see for example [2]); these will be used without reference throughout the rest of the paper. The next preliminary result that we will establish is that metacyclic $p$-groups, $p \neq 2$, are regular.

Lemma 3. Let $G$ be a $p$-group, $p \neq 2$. If $G_{2}$ is cyclic, then $G$ is regular.

Proof. If $g, h \in G$, let $H=\langle g, h\rangle$. Then $(g h)^{p}=g^{p} h^{p} c d$ where $c \in P\left(H_{2}\right)$ and $d \in H_{p}$ [3]. Since $H_{2}$ is cyclic and $H_{p}$ is a proper subgroup of $H_{2}$, it follows that $c d=f^{p}$ where $f \in H_{2}$. Hence $G$ is regular.

Corollary 1. If $G$ is a metacyclic $p$-group, $p \neq 2$, then $G$ is regular.

Let $G$ be a regular $p$-group. An extremely useful class of automorphisms of $G$ is constructed in

Lemma 4. Let $K \triangleleft G$ and let $a \in G$ be such that $G / K=\langle a K\rangle$ is cyclic of order $p^{n}$. If $x \in \Omega_{n}(Z(K))$, then the mapping $\phi(a, K, x)$ defined by $a^{j} k \phi(a, K, x)=(a x)^{j} k$, where $0 \leqq j<p^{n}$ and $k \in K$, is an automorphism 
of $G$ under which $K$ is elementwise fixed. Furthermore, $|\phi(a, K, x)|$ $=|x|$.

Proof. Since $G$ is regular and $x \in \Omega_{n}(Z(K)),(a x)^{p^{n}}=a^{p^{n}}$. Hence $\phi(a, K, x)=\phi$ is an automorphism of $G$ which leaves $K$ elementwise fixed [5]. Since $a \phi^{s}=a x^{8}$, it follows that $|\phi|=|x|$.

TheOREM. If $p \neq 2$ and $G$ is a noncyclic metacyclic p-group of order greater than $p^{2}$, then $|G|$ divides $|A(G)|$.

Proof. We may assume that $G$ is non-Abelian; indeed by $\mathrm{R}$. Faudree's result [1], we may assume that class $G>2$. Choose $a, b \in G$ such that $H=\langle b\rangle$ and $G / H=\langle a H\rangle$ is cyclic of order $k$. Let $G_{2}=\left\langle b^{l}\right\rangle$ where $l$ is a power of $p$. We may assume that $(a, b)=b^{l}$. Furthermore, $\left|b G_{2}\right|=l$ and since class $G>2,\left|a G_{2}\right|=k$. Let $\left|b^{l}\right|=m$. Then $x^{m} \in Z$ for each $x \in G$ and since $\left(b^{l}\right)^{k}=\left(a^{k}, b\right)=e$, we see that $m \leqq k$. Furthermore, since class $G>2$, it is also true that $l<m$. Let $k=r l$ and let $a^{k}=\left(b^{l}\right)^{s}$ where $1<s \leqq m$.

We note that $I(G)=\left\langle I_{a}, I_{b}\right\rangle$ and hence that $|I(G)|=m^{2}$. Also if $\sigma \in I(G)$ and $g, h \in G$ are such that $g \sigma=g h$, then $h \in\left\langle b^{l}\right\rangle=G_{2}$.

To complete the proof we will consider four cases; in each case we will construct a subgroup $S$ of $A(G)$ such that $|S|=k l m=|G|$.

Case I. $s \geqq r$.

Let $c=b^{-s / r} a$. Then $(c, b)=b^{l},|c|=k, G=\langle b, c\rangle$ and $H \cap\langle c\rangle=E$. Let $K=\left\langle c, b^{l}\right\rangle$. Then $K \triangleleft G$ and $G / K=\langle b K\rangle$ is cyclic of order $l$. Also $c^{m / l} \in Z(K)$ and $\left|c^{m / l}\right|=k l / m \geqq l$. Choose $t$ such that $\left|c^{m t / l}\right|=l$ and let $x=c^{m t / l}$. Then $\phi(b, K, x)=\phi \in A(G),|\phi|=l$. Furthermore, $G / H=\langle c H\rangle$ is cyclic of order $k$ and $\theta\left(c, H, c^{m}\right)=\theta \in A(G)$ with order $k / m$. Since $m>l$, it follows that $\phi \in C(\langle\theta\rangle)$. Hence, if $S=\langle\phi, \theta, I(G)\rangle$, then $|S|=k l m$.

Case II. $1<s<r, k / s \leqq m$.

Let $d=a^{-r / s} b$. Then $(a, d)=b^{l},|d|=l s \geqq m, G=\langle a, d\rangle$ and $H \cap\langle d\rangle=E$. Let $L=\left\langle a, b^{l}\right\rangle$. Then $L \triangleleft G$ and $G / L=\langle b L\rangle=\langle d L\rangle$ is cyclic of order $l$. Finally, if $M=\left\langle d, b^{l}\right\rangle$, then $M \triangleleft G$ and $G / M=\langle a M\rangle$ is cyclic of order $k / s$. We note that $\left(d^{m s / k}, b^{l}\right)=e$ and that $\left|d^{m s / k}\right|=k l / m \geqq k / s$. If we choose $u$ such that $\left|d^{m s u / l}\right|=k / s$ and let $y=d^{m s u / l}$, then $\phi(a, M, y)$ $=\phi \in A(G)$ and $|\phi|=k / s$. Furthermore, since $l s \leqq l m, \theta\left(d, L, d^{m}\right)$ $=\theta \in A(G)$ with order $l s / m$. Since $k / s \leqq m$, it follows that $\phi \in C(\langle\theta\rangle)$. Thus if $S=\langle\phi, \theta, I(G)\rangle,|S|=k l m$.

Case III. $1<s<r, k / s>m, l s \leqq k / s$.

Since $k / s>m,\left(d, b^{l}\right)=e$. Thus $d \in \Omega_{k / s}(Z(M))$ and $\phi(a, M, d)=\phi$ $\in A(G)$ with order $l s$. If $R=\langle\phi, I(G)\rangle$, then $|R|=l s m^{2}$. Furthermore, since $|a|=k m / s, \theta\left(a, M, a^{m}\right)=\theta \in A(G)$ and $|\theta|=k / s$. Since 
$\theta \in N(\langle\phi\rangle)$, if $S=\langle\theta, R\rangle$, then $|S|=|R| \cdot[S: R]$. By Lemma $1,[S: R]$ $=\left|a^{m} M\right|=k / m s$. Hence $|S|=k l m$.

Case IV. $1<s<r, k / s>m, l s>k / s$.

Choose $v$ such that $l s=k v / s$. Then $d^{v} \in \Omega_{k / s}(Z(M))$ and $\phi\left(a, M, d^{v}\right)$ $=\phi \in A(G)$ with order $k / s$. Also $\theta\left(d, L, d^{m}\right)=\theta \in A(G),|\theta|=l s / m$ and $\theta \in N(\langle\phi\rangle)$. Finally, letting $S=\langle\phi, \theta, I(G)\rangle$, we see that $|S|=k l m$ and the proof of the theorem is complete.

\section{REFERENCES}

1. R. Faudree, $A$ note on the automorphism group of a p-group, Proc. Amer. Math. Soc. 19 (1968), 1379-1382.

2. M. Hall, Jr., The theory of groups, Macmillan, New York, 1959. MR 21 \#1996.

3. P. Hall, A contribution to the theory of groups of prime-power orders, Proc. London Math. Soc. (2) 36 (1933), 29-95.

4. J. C. Howarth, Some automorphisms of finite nilpotent groups, Proc. Glasgow Math. Assoc. 4 (1960), 204-207. MR 22 \#9537.

5. O. J. Huval, A note on the outer automorphisms of finite nilpotent groups, Amer. Math. Monthly 73 (1966), 174-175.

6. A. D. Otto, Central automorphisms of a finite p-group, Trans. Amer. Math. Soc. 125 (1966), 280-287. MR 34 \#4362.

Lafayette College, Easton, Pennsylvania 18042 